\title{
High salt medium activates RhoA/ROCK and downregulates eNOS expression via the upregulation of ADMA
}

\author{
$\mathrm{YU} \mathrm{CAO}^{1}$, YUAN FANG ${ }^{2}$, JIANJUN MU ${ }^{2}$ and XIAOHONG LIU ${ }^{1}$ \\ Departments of ${ }^{1}$ Pediatrics and ${ }^{2}$ Cardiovascular Medicine, \\ First Affiliated Hospital of Medical College of Xi'an Jiaotong University, Xi'an, Shaanxi 710061, P.R. China
}

Received June 2, 2015; Accepted April 18, 2016

DOI: $10.3892 / \mathrm{mmr} .2016 .5241$

\begin{abstract}
Endothelial dysfunction has an important role in the development and progression of salt-sensitive hypertension. Asymmetric dimethylarginine (ADMA), which is an endogenous inhibitor of nitric oxide synthase (NOS), has been demonstrated to be involved in the pathophysiological processes of endothelial dysfunction and salt-sensitive hypertension. However, it is currently unclear how high salt intake may induce these processes. The present study investigated the effects of high salt medium on ADMA, endothelial NOS (eNOS) and the Ras homolog gene family, member A (RhoA)/Rho-associated protein kinase (ROCK) pathway in the EA.hy926 umbilical vein cell line. The results demonstrated that high salt medium significantly increased the concentration of ADMA, the expression of protein arginine methyltransferase 1 (PRMT-1) and RhoA, and the activity of ROCK, and downregulated the expression of eNOS. Knockdown of PRMT-1 with small interfering RNA (siRNA) significantly abrogated the aforementioned effects. These results indicated that ADMA has a key role in high salt-mediated activation of the RhoA/ROCK pathway and inhibition of eNOS biosynthesis. siRNA-PRMT-1 may be considered a novel remedy for the treatment of endothelial dysfunction.
\end{abstract}

\section{Introduction}

Endothelial dysfunction is one of the most important mechanisms underlying the development and progression of salt-induced hypertension (1). Reduced bioavailability of nitric oxide $(\mathrm{NO})$ in the vasculature is a major feature of endothelial dysfunction, which leads to increased endothelial permeability, platelet aggregation, leukocyte adhesion, and

Correspondence to: Dr Yuan Fang, Department of Cardiovascular Medicine, First Affiliated Hospital of Medical College of Xi'an Jiaotong University, 277 Yanta West Road, Xi'an, Shaanxi 710061, P.R. China

E-mail: fangyuanwbt@163.com

Key words: endothelial dysfunction, asymmetric dimethylarginine, small interfering RNA, endothelial nitric oxide synthase cytokine generation $(2,3)$. Endothelial NO synthase (eNOS) is the predominant NOS isoform, which is responsible for generating the majority of $\mathrm{NO}$ in the vasculature. Reductions in the expression or activity of eNOS, and its uncoupling, have a crucial role in the process of endothelial dysfunction (4). Asymmetric dimethylarginine (ADMA) is an endogenous inhibitor of NOS, the dysregulation of which is associated with endothelial dysfunction and salt-sensitive hypertension (5-7).

The small GTPase Ras homolog gene family, member A (RhoA) anditsdownstream target Rho-associated proteinkinase (ROCK) are important in the regulation of endothelial barrier function. The RhoA/ROCK pathway regulates cell motility, migration, proliferation and angiogenesis via the control of actin-cytoskeletal assembly and cell contraction (8). Furthermore, it has previously been demonstrated that the RhoA/ROCK pathway has a central role in impaired production of NO, due to its numerous actions on eNOS (9-11).

ADMA is synthesized endogenously during the methylation of protein arginine residues by protein arginine methyltransferases (PRMTs). PRMTs are a protein family that monomethylate or dimethylate the guanidino nitrogen atoms on arginine side chains. PRMT-1 is the predominant type I PRMT in mammalian cells, which catalyzes arginine residues facilitating the formation of ADMA residues (12). In the cardiovascular system, ADMA is generated by PRMT-1, which is expressed in the heart, and smooth muscle and endothelial cells. It has previously been reported that altering ADMA metabolism in vivo and in vitro may affect the activity of Rho GTPases, which are key regulators of actin dynamics, endothelial cell motility and angiogenesis (13). The plasma levels of ADMA are elevated concomitantly with increased ROCK activity and ROCK 1 expression in the pulmonary arteries of Sprague-Dawley rats (14). In addition, in pulmonary endothelial cells, exogenous and endogenous ADMA increases RhoA activity $(15,16)$.

Animal and population studies have demonstrated that high salt intake can induce a small increase (2-4 mmol/l) in plasma sodium levels, which may influence blood pressure $(17,18)$. High salt intake may result in hypertension and cardiovascular complications via its effects on signals triggered by augmented extracellular $\mathrm{NaCl}$ concentration and/or osmolality of extracellular fluids (19). Endothelial cells, as vascular salt sensors, are highly sensitive to extracellular sodium (20). The present study tested the hypothesis that high salt medium could increase 
the secretion of ADMA, further upregulate RhoA/ROCK expression and activity, and that downregulation of ADMA by RNA interference (RNAi) could significantly reverse these processes.

\section{Materials and methods}

Cell culture. The EA.hy926 cells (Cell Bank of the Chinese Academy of Sciences, Shanghai, China) were maintained in high glucose Dulbecco's modified Eagle's medium (DMEM; Hyclone; GE Healthcare Life Sciences, Logan, UT, USA) supplemented with $10 \%$ fetal bovine serum (Hyclone; GE Healthcare Life Sciences). The cells were cultured at $37^{\circ} \mathrm{C}$ in an atmosphere containing $5 \% \mathrm{CO}_{2}$ and $95 \%$ air, and were subcultured by trypsinization every $36-48 \mathrm{~h}$. Cells from the $5^{\text {th }}-10^{\text {th }}$ passage were used in subsequent experiments.

Cell viability assay. To determine the optimum concentration of high salt medium on EA.hy926 cells, the cells were treated with serum-free DMEM (109 mmol/l, which contained the standard concentration of $\mathrm{NaCl}$ ) as a control; with high salt medium at various concentrations of salinity $(115,130$, 137, 147 and $160 \mathrm{mmol} / \mathrm{l}$ ); or with isotonic mannitol (solid mannitol diluted in DMEM) at 12 or $56 \mathrm{mmol} / \mathrm{l}$ as a hyperosmotic control, for $48 \mathrm{~h}$. Cell viability was determined using WST-8 dye (Beyotime Institute of Biotechnology, Haimen, China) according to manufacturer's protocol. A total of $5 \times 10^{3}$ cells/well $(100 \mu \mathrm{l})$ were seeded into a 96-well plate, and were incubated at $37^{\circ} \mathrm{C}$ for $48 \mathrm{~h}$. Subsequently, the cells were treated with the aforementioned media for $24 \mathrm{~h}$, each group consisted of four wells. WST-8 dye solution $(10 \mu \mathrm{l})$ was then added to each well, and the cells were incubated at $37^{\circ} \mathrm{C}$ for a further $2 \mathrm{~h}$. Absorbance (A) was finally determined by spectrophotometry at $560 \mathrm{~nm}$ using a microplate reader (BC Biotech Engineering Ltd., London, UK). Cell viability was calculated using the following equation: Cell viability $=\left(\mathrm{A}_{450}\right.$ of treatment wells - $\mathrm{A}_{450}$ of control wells) / $\mathrm{A}_{450}$ of control wells $x 100 \%$. All data were expressed as the mean \pm standard error of the mean of three experiments.

PRMT-1 knockdown with RNAi. Three PRMT-1-specific small interfering RNAs (siRNAs), negative and positive control siRNAs were designed and synthesized by Shanghai GeneChem Co., Ltd. (Shanghai, China). To optimize transfection, various concentrations of siRNA $(25,50,100$ and $150 \mathrm{nM}$ ) in serum-free DMEM were transfected into the cells in six-well plates using TurboFect siRNA Transfection Reagent (Fermentas; Thermo Fisher Scientific, Inc., Pittsburgh, PA, USA) according to the manufacturer's protocol. Cells were collected $48 \mathrm{~h}$ post-transfection to assess gene silencing using reverse transcription-quantitative polymerase chain reaction (RT-qPCR). The siRNA for PRMT-1 (siPRMT1-1 ${ }^{\#}$ ) at the final concentration of $50 \mathrm{nM}$ was found to be the most efficient at knocking down gene expression, with minimal toxicity. The sequence is as follows: Sense, 5'-CCAUCGACCUGGACU UCAATT-3' and antisense, 5'-UUGAAGUCCAGGUCGAUG GTT-3'.

Cell treatment. Once the cell monolayers reached $80 \%$ confluence, the cells were incubated with serum-free medium for
$16 \mathrm{~h}$, and were then treated with various concentrations of high salt medium. The cells were treated with DMEM; high salt medium (115 or $137 \mathrm{mmol} / \mathrm{l})$; high salt medium (115 mmol/l) and siRNA-PRMT-1 (50 nM); high salt medium (137 mmol/l) and siRNA-PRMT-1 (50 nM); or with isotonic mannitol (12 or $56 \mathrm{mmol} / \mathrm{l}$ ) as an osmotic control, for $48 \mathrm{~h}$, after which they were harvested for further analysis.

Measurement of ADMA in culture supernatant. Following a $48 \mathrm{~h}$ incubation, the medium was transferred to microcentrifuge tubes and was centrifuged at $10,000 \mathrm{x}$ g for $10 \mathrm{~min}$ at $4^{\circ} \mathrm{C}$. The concentration of ADMA was determined by high-performance liquid chromatography. To prepare samples, the supernatant $(100 \mu \mathrm{l})$ was diluted with $0.1 \mathrm{~mol} / \mathrm{l}$ hydrochloric acid to $1.5 \mathrm{ml}$. The samples $(20 \mu \mathrm{l})$ and standards $(20 \mu \mathrm{l})$ were incubated for $3 \mathrm{~min}$ with $o$-phthaldialdehyde reagent $(10 \mathrm{mg} / \mathrm{mL}$ OPA in borate buffer, $\mathrm{pH} 9.0$, containing $0.4 \%$ mercaptoethanol. ADMA and $o$-phthaldialdehyde were purchased from Sigma-Aldrich (St. Louis, MO, USA). Briefly, a Shimadzu LC-10A liquid chromatograph equipped with a Model 7125i injection valve and a Shimadzu RF-10AXL fluorescence detector was used (Shimadzu Corporation, Kyoto, Japan). N2010 (Zhejiang University, Hangzhou, China) was used as a data processor. A $5 \mu \mathrm{M}$ Waters Symmetry C18 $(5 \mu \mathrm{m}$; $150 \times 3.9 \mathrm{~mm}$ ) coupled to a Waters Sentry Symmetry C18 guard column (5 $\mu \mathrm{m} ; 3.9 \times 20 \mathrm{~mm})$ (Waters UK, Elstree, UK) was operated at room temperature. The mobile phase consisted of 77:23 (v/v) potassium phosphate-buffer $(\mathrm{pH} 3.5)$ :acetonitrile. The flow rate was $1.0 \mathrm{ml} / \mathrm{min}$. The injection volume was $20 \mu \mathrm{l}$. $o$-Phthaldialdehyde adducts of methylated amino acids and internal standard ADMA produced by pre-column mixing were monitored at 338 and $447 \mathrm{~nm}$, respectively.

RNA isolation and RT-qPCR. Total RNA was isolated from the cells using TRIzol ${ }^{\circledR}$ reagent (Invitrogen; Thermo Fisher Scientific, Inc., Waltham, MA, USA). cDNA was synthesized from RNA using the RevertAid ${ }^{\text {TM }}$ First strand cDNA Synthesis kit (Fermentas; Thermo Fisher Scientific, Inc.). Briefly, the RNA/primer mixture was placed into a sterile, nuclease free tube on ice; $4 \mu \mathrm{l}$ 5X Reaction Buffer, $1 \mu \mathrm{l}$ Ribolock RNase inhibitor, $2 \mu 110 \mathrm{mM}$ dNTP mix and Reverse Transcriptase were mixed and centrifuged at $10,00 \mathrm{x} \mathrm{g}$, and incubated for $60 \mathrm{~min}$ at $42^{\circ} \mathrm{C}$. The reaction was terminated by heating at $70^{\circ} \mathrm{C}$ for $5 \mathrm{~min}$. The mRNA expression levels of PRMT-1, eNOS and RhoA were detected by qPCR, which was performed on an iQ5 Real-Time PCR Detection system (Bio-Rad Laboratories, Inc., Hercules, CA, USA) using SYBR ${ }^{\circledR}$ Premix Ex Taq ${ }^{\mathrm{TM}}$ II (Takara Bio, Inc., Shiga, Japan). The reaction volume was

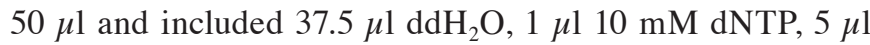
10X PCR buffer, $3 \mu 125 \mathrm{mM} \mathrm{MgCl}{ }_{2}, 1 \mu \mathrm{l}$ forward primer, $1 \mu \mathrm{l}$ reverse primer and $1 \mu \mathrm{l} \mathrm{cDNA}$. The thermocycling conditions were as follows: $3 \mathrm{~min}$ at $95^{\circ} \mathrm{C}$, followed by 40 cycles at $95^{\circ} \mathrm{C}$ for $10 \mathrm{sec}, 58^{\circ} \mathrm{C}$ for $30 \mathrm{sec}$ and $72^{\circ} \mathrm{C}$ for $30 \mathrm{sec}$. The data were collected at the annealing step $\left(72^{\circ} \mathrm{C}\right)$ of each cycle. The primer sequences (Sangon, Shanghai, China) are presented in Table I. Relative gene expression levels were normalized to glyceraldehyde 3-phosphate dehydrogenase (GAPDH). Data were analyzed on the basis of the relative expression method, using the $2^{-\Delta \Delta \mathrm{Cq}}$ relative expression formula, where $\Delta \Delta \mathrm{Cq}=\Delta \mathrm{Cq}$ (experimental group) $-\Delta \mathrm{Cq}$ (control group), 
Table I. Primer sequences for reverse transcription-quantitative polymerase chain reaction.

\begin{tabular}{ll}
\hline Gene & \multicolumn{1}{c}{ Sequence } \\
\hline GAPDH & F: ATCGTGCGTGACATTAAGGAGAAG \\
& R: AGGAAGGAAGGCTGGAAGAGAG \\
PRMT-1 & F: GCCTCCAGCCGCCCTCTTG \\
& R: CACCTCGTCCTTCAGCATCTCC \\
eNOS & F: CACCGCTACAACATCCAG \\
& R: GCCTTCTGCTCATTCTCC \\
RhoA & F: TGCTTGCTCATAGTCTTCAG \\
& R:CACATCAGTATAACATCGGTATC
\end{tabular}

GAPDH, glyceraldehyde 3-phosphate dehydrogenase; PRMT-1, protein arginine methyltransferase 1; eNOS, endothelial nitric oxide synthase; RhoA, Ras homolog gene family, member A; F, forward; $\mathrm{R}$, reverse.

$\Delta \mathrm{Cq}=\mathrm{Cq}$ (target gene) $-\mathrm{Cq}(\mathrm{GAPDH})$, and $\mathrm{Cq}$ is the cycle at which the threshold is crossed (21).

Western blot analysis. The cells were homogenized and lysed with radioimmunoprecipitation assay lysis buffer (GSE Bio, Shaanxi, China). The supernatant was collected following centrifugation at $10,000 \mathrm{x}$ g for $15 \mathrm{~min}$ at $4^{\circ} \mathrm{C}$. Proteins in the supernatant were quantified by the Bradford assay using a protein assay kit. Equal amounts of protein $(45 \mu \mathrm{g})$ were separated by $12 \%$ sodium dodecyl sulfate-polyacrylamide gel electrophoresis, and were transferred to nitrocellulose membranes (EMD Millipore, Billerica, MA, USA) at $250 \mathrm{~mA}$ for $2 \mathrm{~h}$. Non-specific binding was blocked with 5\% skim milk for $2 \mathrm{~h}$ at room temperature. Immunoblotting was then performed using PRMT-1 (mouse monoclonal; cat. no. ab12189; Abcam, Cambridge, UK); eNOS (mouse monoclonal; cat. no. 9572), RhoA (rabbit monoclonal; cat. no. 2117), phosphorylated (p)-myosin phosphatase target subunit 1 (MYPT-1; rabbit monoclonal; cat. no. 5163) and total MYPT-1 (rabbit monoclonal; cat. no. 2634) (all 1:1,000; Cell Signaling Technology, Inc., Beverly, MA, USA); and GAPDH (rabbit monoclonal; 1:5,000; cat. no. AP0063; Bioworld Technology, Inc., St. Louis Park, MN, USA) antibodies at $4^{\circ} \mathrm{C}$ overnight. The membranes were then washed and probed with horseradish peroxidase-conjugated goat anti-rabbit (cat. no. 31460), goat anti-rat (cat. no. 31430) (both 1:5,000; Thermo Fisher Scientific, Inc., Waltham, MA, USA); and mouse anti-goat (1:1,000; cat. no. bse-0294M; Beijing Biosynthesis Biotechnology Co., Ltd., Beijing, China) antibodies for $1 \mathrm{~h}$ at room temperature. Subsequently, the membranes were washed with Tris-buffered saline containing $0.1 \%$ Tween. Densitometric analysis was performed using Bio-Rad iQ5 image software (Bio-Rad Laboratories, Inc.), and the ratio relative to GAPDH expression was calculated for each sample. Blots were visualized using chemiluminescent solution (GSE Bio). ROCK activity was assessed as the relative ratio of p-MYPT-1/total MYPT-1.

Statistical analysis. All experiments were performed in triplicate. Data are presented as the mean \pm standard error

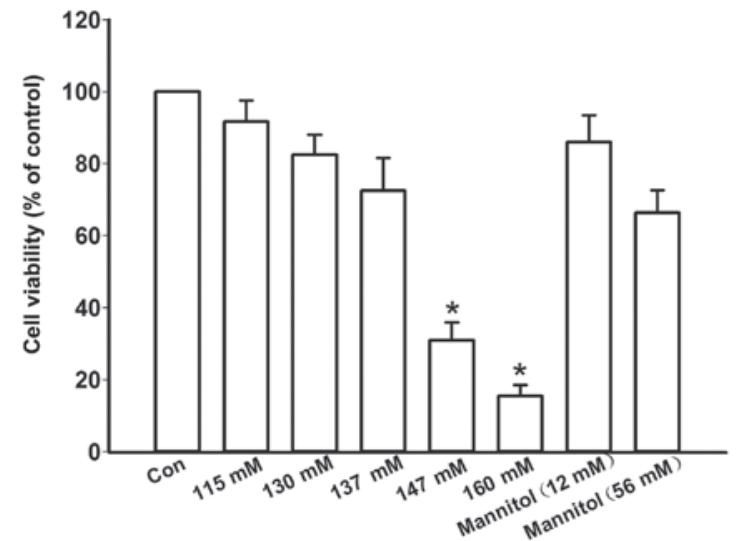

Figure 1. Effects of various treatments on the viability of EA.hy926 cells, as determined by WST- 8 assay. Cells were treated with Dulbecco's modified Eagle's medium (Con), various concentrations of high salt medium, or with mannitol as an osmotic control. Con, normal control group. ${ }^{*} \mathrm{P}<0.05$ compared with the Con group.

of the mean. Differences between the treatment groups were compared by unpaired t-test or one-way analysis of variance, followed by the Student-Newman-Keuls post-hoc test for multiple comparisons. $\mathrm{P}<0.05$ was considered to indicate a statistically significant difference.

\section{Results}

Effects of high salt medium on cell viability. In order to determine the optimal high salt concentration without affecting cell viability, the cells were initially treated with high salt medium containing various concentration of $\mathrm{NaCl}$, or with mannitol as an osmotic control. High salt medium at a concentration of $147 \mathrm{mM}(30.1 \pm 5.0 \%)$ and $160 \mathrm{mM}$ $(15.5 \pm 3.1 \%)$ significantly decreased cell viability compared with in the control group $(\mathrm{P}=0.01$ and $\mathrm{P}<0.001$, respectively; Fig. 1). High salt medium at a concentration of 115,130 and $137 \mathrm{mM}$ caused a slight decrease in cell viability; however, the difference was statistically insignificant among the groups $(\mathrm{P}=0.07, \mathrm{P}=0.06$ and $\mathrm{P}=0.06$, respectively, Fig. 1$)$. Therefore, 115 and $137 \mathrm{mmol} / \mathrm{l}$ concentrations were chosen to treat the cells, in order to observe the effects of high salt medium.

Optimization of siRNA-PRMT-1 cell transfection. RNAi was used to knockdown the expression of PRMT-1, which synthesizes ADMA. After $48 \mathrm{~h}$, the gene silencing efficiency of siRNA-PRMT-1 was detected using RT-qPCR. The mRNA expression levels of PRMT-1 in the $25 \mathrm{nM}$ group $(0.28 \pm 0.02)$ were significantly higher than in the $50 \mathrm{nM}(0.18 \pm 0.03 ; \mathrm{P}=0.03)$, $100 \mathrm{nM}(0.15 \pm 0.02 ; \mathrm{P}=0.03)$ and $150 \mathrm{nM}$ groups $(0.13 \pm 0.02$; $\mathrm{P}=0.02$ ) (Fig. 2). Transfection with $50 \mathrm{nM}$ siRNA-PRMT-1 induced a five-fold reduction in PRMT-1 mRNA expression compared with the control group (1.2 $\pm 0.06 ; \mathrm{P}=0.01$; Fig. 2$)$. Since high concentrations of siRNA may exhibit cytotoxicity, $50 \mathrm{nM}$ was selected as the concentration of siRNA used in subsequent experiments.

Effects of various treatments on ADMA concentration. The cells were treated with high salt medium for $48 \mathrm{~h}$, and the 


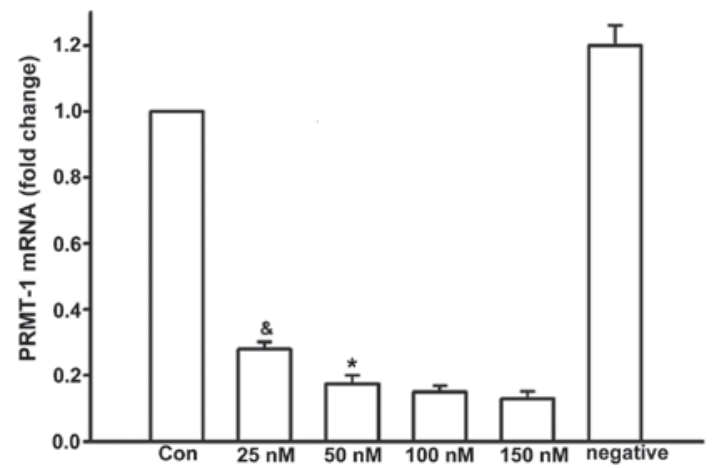

Figure 2. Effects of various siRNA concentrations on the mRNA expression of PRMT-1. siRNA, small interfering RNA; PRMT-1, protein arginine methyltransferase 1; Con, untransfected control group; negative, negative control group. ${ }^{\&} \mathrm{P}<0.05$, compared with the three other siRNA transfection groups; ${ }^{*} \mathrm{P}<0.05$, compared with the control group.

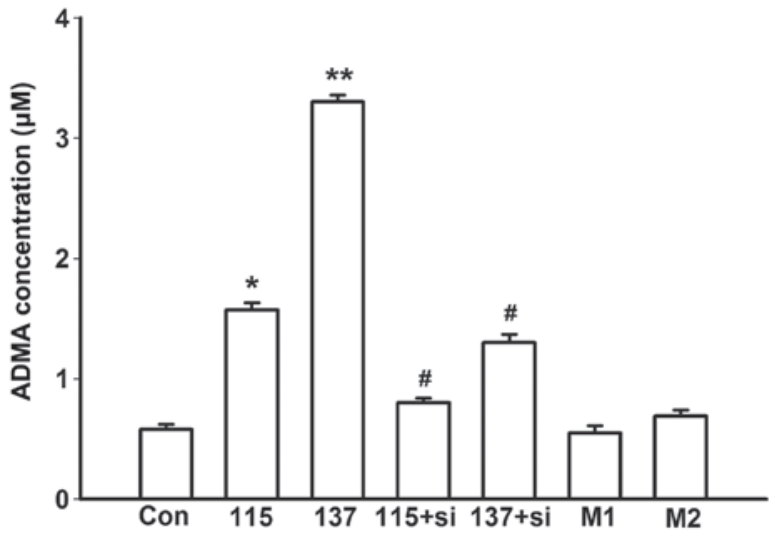

Figure 3. Effects of various treatments on the concentration of ADMA in culture supernatants. Con, control group; $115+\mathrm{si}, 115 \mathrm{mmol} / 1$ high salt medium treatment and $50 \mathrm{nM}$ siRNA-PRMT-1 transfection; 137+si, $137 \mathrm{mmol} / 1$ high salt medium treatment and $50 \mathrm{nM}$ siRNA-PRMT-1 transfection; M1, 12 mmol/1 mannitol; M2, 56 mmol/1 mannitol; siRNA, small interfering RNA; PRMT-1, protein arginine methyltransferase $1 .{ }^{*} \mathrm{P}<0.05$ compared with the Con group; ${ }^{* *} \mathrm{P}<0.05$ compared with all other groups; ${ }^{*} \mathrm{P}<0.05$ compared with the high salt medium-treated untransfected groups.

concentration of ADMA was measured in the culture supernatants. Treatment with high salt medium induced a marked increase in ADMA in a concentration-dependent manner (115 vs. Con, $\mathrm{P}=0.01 ; 137$ vs. Con, $\mathrm{P}<0.001$; Fig. 3 ). The increased concentration of ADMA was verified to be induced by high salt since there was no change in ADMA concentration in the mannitol osmotic control groups (M1 vs. Con, $\mathrm{P}=0.17$; $\mathrm{M} 2$ vs. Con, $\mathrm{P}=0.25$; Fig. 3).

Subsequently, RNAi was used to knockdown PRMT-1 expression, and the cells were treated with high salt medium for $48 \mathrm{~h}$, prior to measurement of ADMA concentration in the supernatants. ADMA concentration in the siRNA-transfected (50 nM) groups was significantly decreased compared with in the untransfected groups $(115+\mathrm{si}$ vs. $115, \mathrm{P}=0.03 ; 137+\mathrm{si}$ vs. 137, P=0.01; Fig. 3). Knockdown of PRMT-1 almost abrogated the effects of high salt medium on ADMA secretion. These results indicate that salt levels, but not osmotic pressure, in high salt medium have an effect on ADMA biosynthesis (Fig. 3).
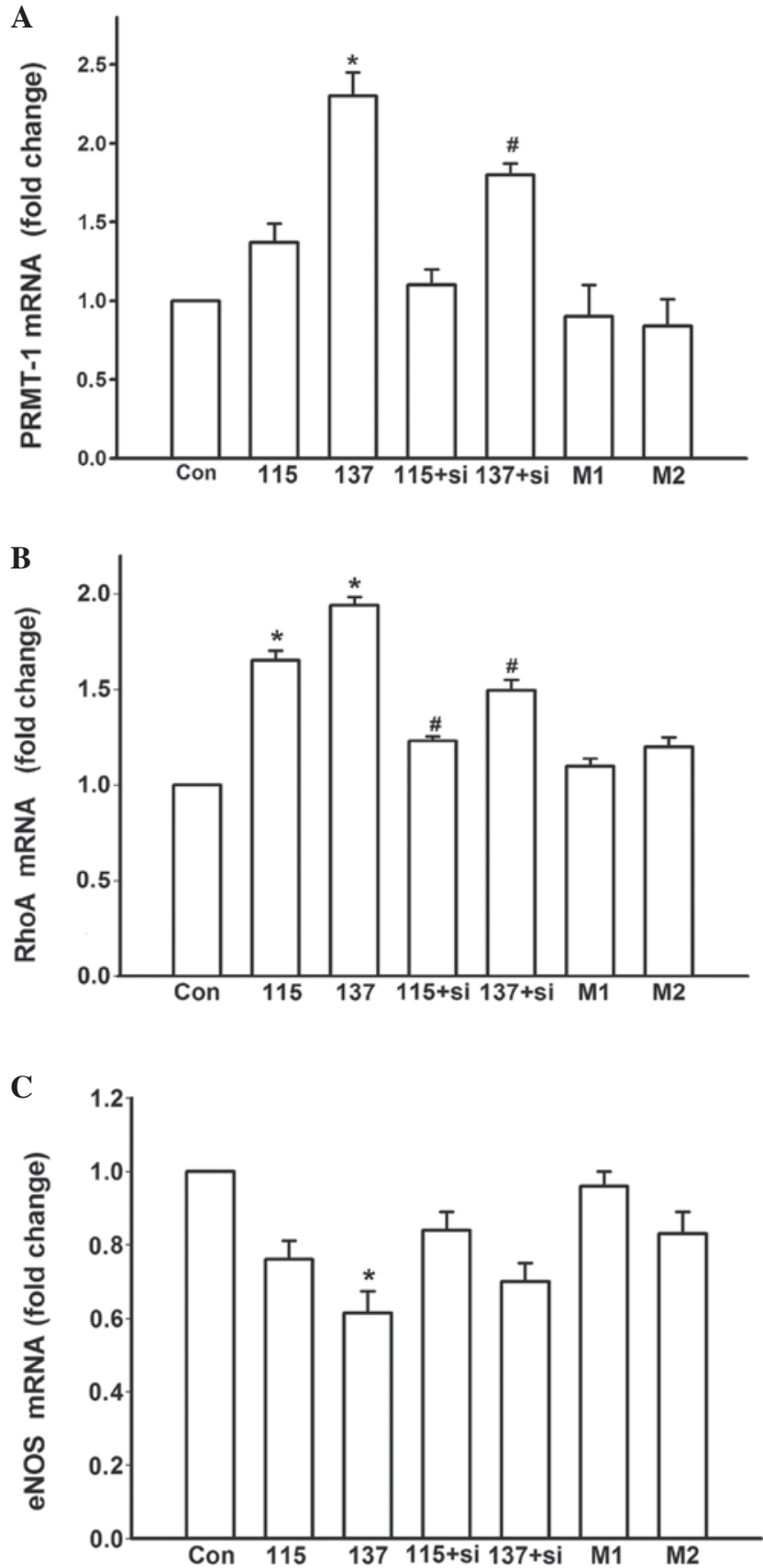

Figure 4. Effects of various treatments on the mRNA expression levels of PRMT-1, RhoA and eNOS after a 48 h incubation. Relative (A) PRMT-1, (B) RhoA and (C) eNOS mRNA expression. Con, control group; 115+si, $115 \mathrm{mmol} / 1$ high salt medium treatment and $50 \mathrm{nM}$ siRNA-PRMT-1 transfection; $137+\mathrm{si}, 137 \mathrm{mmol} / 1$ high salt medium treatment and $50 \mathrm{nM}$ siRNA-PRMT-1 transfection; M1, 12 mmol/1 mannitol; M2, 56 mmol/1 mannitol; si, small interfering RNA; PRMT-1, protein arginine methyltransferase 1; eNOS, endothelial nitric oxide synthase; RhoA, Ras homolog gene family, member $\mathrm{A}$. ${ }^{*} \mathrm{P}<0.05$ compared with the Con group; ${ }^{\#} \mathrm{P}<0.05$ compared with the high salt medium-treated untransfected groups.

Effects of various treatments on the $m R N A$ and protein expression levels of PRMT-1, eNOS and RhoA, and ROCK activity. In order to determine whether high salt treatment was able to alter the expression of PRMT-1, eNOS, RhoA and ROCK, the cells were cultured in high salt medium for $48 \mathrm{~h}$ and RNA and protein were extracted (Figs. 4 and 5). mRNA expression levels were quantified using RT-qPCR. Treatment with high salt medium significantly increased the mRNA 


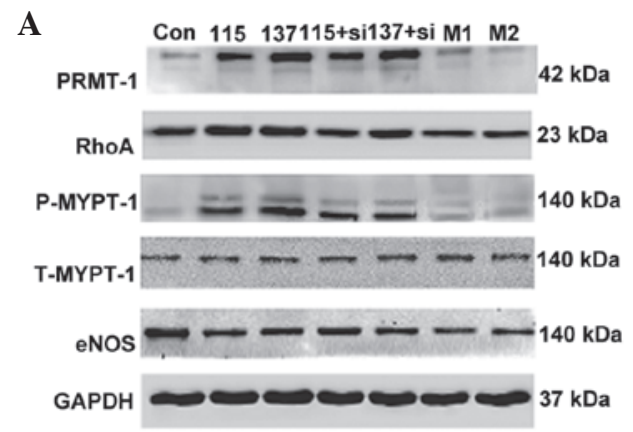

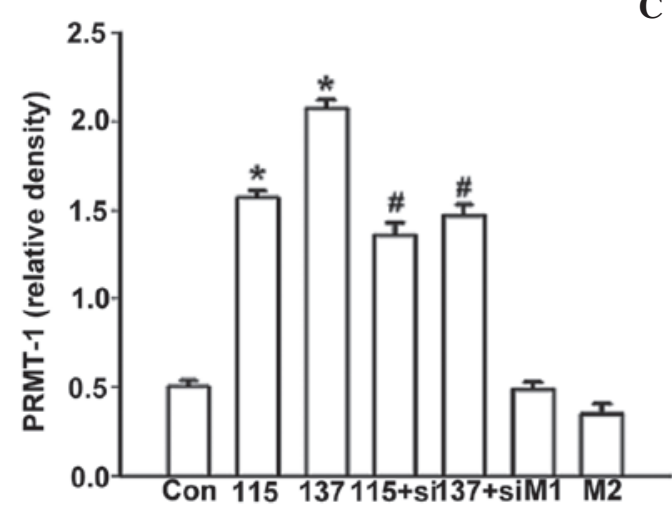

C

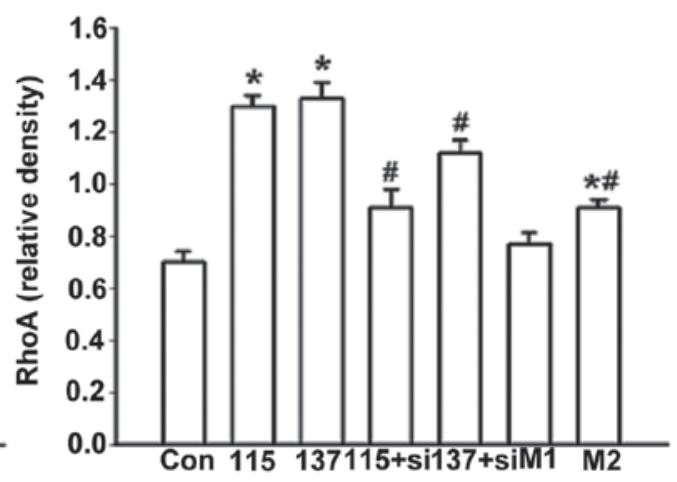

D

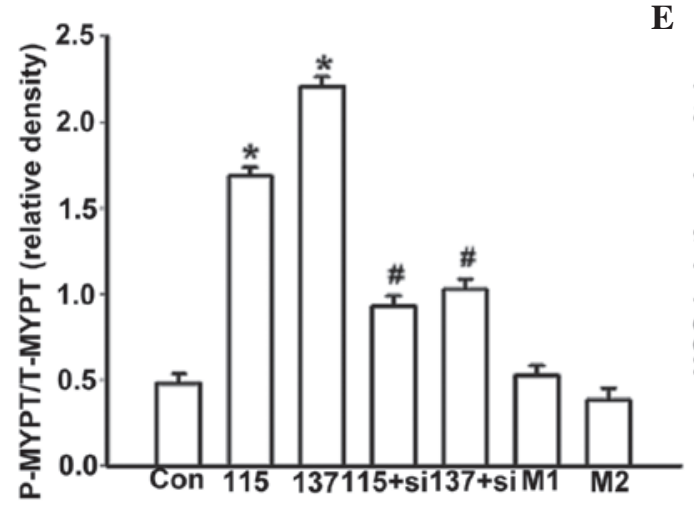

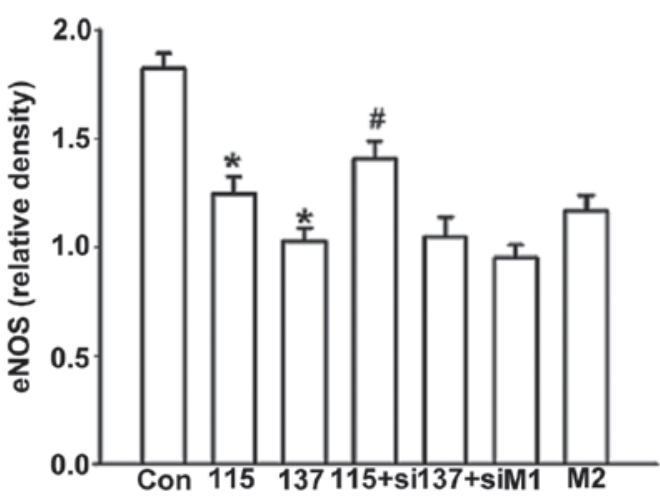

Figure 5. Effects of various treatments on the protein expression of PRMT-1, eNOS, RhoA and ROCK after $48 \mathrm{~h}$. (A) Western blot analysis was conducted to detect the protein expression levels of PRMT-1, RhoA, p-MYPT-1, total MYPT-1, eNOS and GAPDH. Relative quantification of (B) PRMT-1 protein expression; (C) RhoA protein expression; (D) ROCK activity and (E) eNOS protein expression. Con, control group; $115+s i, 115 \mathrm{mmol} / 1$ high salt medium treatment and $50 \mathrm{nM}$ siRNA-PRMT-1 transfection; 137+si, $137 \mathrm{mmol} / 1$ high salt medium treatment and $50 \mathrm{nM}$ siRNA-PRMT-1 transfection; M1, $12 \mathrm{mmol} / 1 \mathrm{mannitol}$; M2, 56 mmol/1 mannitol; si, small interfering RNA;PRMT-1, protein arginine methyltransferase 1; eNOS, endothelial nitric oxide synthase; RhoA, Ras homolog gene family, member A; ROCK Rho-associated protein kinase. ${ }^{\mathrm{P}}<0.05$ compared with the Con group; ${ }^{\text {" }} \mathrm{P}<0.05$ compared with the high salt medium-treated untransfected groups.

expression levels of PRMT-1 (137 vs. Con, $\mathrm{P}=0.02$; Fig. 4A) and RhoA (115 vs. Con, $\mathrm{P}=0.01 ; 137$ vs. Con, $\mathrm{P}=0.01$; Fig. 4B), and partially inhibited the expression of eNOS (137 vs. Con, $\mathrm{P}=0.01$; Fig. $4 \mathrm{C}$ ). The same trend was detected at the protein level (Fig. 5A-C and E). The ratio of p-MYPT-1/total MYPT-1 (Thr 696) was used to determine RhoA/ROCK activity (22). ROCK activity was significantly elevated in response to high salt medium compared with the control group (115 vs. Con, $\mathrm{P}=0.02 ; 137$ vs. Con, $\mathrm{P}=0.01$; Fig. 5D). Treatment with mannitol (56 mmol/l) significantly enhanced the protein expression of RhoA but to a lower extent than high salt medium treatment (M2 vs. Con, $\mathrm{P}=0.04$; $\mathrm{M} 2$ vs. 137, $\mathrm{P}=0.03$; Fig. 5C). Furthermore, high salt medium, but not osmotic pressure, significantly increased MYPT-1 phosphorylation (115 vs. Con, $\mathrm{P}=0.02 ; 137$ vs. Con, $\mathrm{P}=0.01$; Fig. 5D).

To explore the potential signaling pathways, cells were treated with siRNA-PRMT-1 to inhibit ADMA production. The aforementioned high salt-induced alterations of gene expression were significantly inhibited following PRMT-1 knockdown $(137+$ si vs. $137, \mathrm{P}=0.02$, Fig. $4 \mathrm{~A} ; 115+$ si vs. 115 , $\mathrm{P}=0.03$; $137+$ si vs. $137, \mathrm{P}=0.02$, Fig. $4 \mathrm{~B} ; 115+$ si vs. $115, \mathrm{P}=0.03$; 137+si vs. 137, $\mathrm{P}=0.02$, Fig. 4B; $115+$ si vs. $115, \mathrm{P}=0.04 ; 137+$ si vs. $137, \mathrm{P}=0.02$, Fig. $5 \mathrm{~B}$; $115+$ si vs. $115, \mathrm{P}=0.02$, Fig. $5 \mathrm{E}$ ). Specific PRMT-1 RNAi inhibited RhoA/ROCK activity (115+si vs. $115, \mathrm{P}=0.02 ; 137+$ si vs. $137, \mathrm{P}=0.03$, Fig. $5 \mathrm{C} ; 115+\mathrm{si}$ vs. $115, \mathrm{P}=0.01 ; 137+$ si vs. $137, \mathrm{P}=0.01$, Fig. $5 \mathrm{D})$. These results 
strongly indicate that to some extent, high salt intake may regulate the expression of RhoA and eNOS via downregulation of ADMA.

\section{Discussion}

The present study is the first, to the best of our knowledge, to demonstrate that high salt medium significantly upregulates ADMA concentration, PRMT-1 and RhoA expression, and ROCK activity, and downregulates eNOS expression in EA.hy926 cells. In addition, these effects could be partially attenuated when PRMT-1 expression was silenced by RNAi.

Previous studies have reported that plasma ADMA levels are increased after salt loading and decreased after salt restriction in salt-sensitive subjects $(7,23,24)$. Animal experiments have also demonstrated that salt loading induces an acute increase in oxidative stress, which contributes to salt-sensitive hypertension $(25,26)$. Furthermore, elevation of $\mathrm{NaCl}$ induces oxidative stress and ROS elevation in vitro (27-29). The activities of PRMTs and eNOS have previously been revealed to be regulated in a redox-sensitive fashion (30). ROS and ADMA are able to form a tightly coupled amplification system, thus aggravating the pathological progression of endothelial dysfunction. The present study demonstrated that the expression levels of PRMT-1 and ADMA were elevated in response to high salt medium in a dose-dependent manner. Therefore, it may be hypothesized that high salt medium induces oxidative stress and stimulates ADMA production via the upregulation of PRMT-1.

Hypertonic stress induces activation of the RhoA/ROCK signaling pathway in cells $(31,32)$. These previous findings are consistent with the findings of the present study, which demonstrated that high salt medium and mannitol $(56 \mathrm{mmol} / \mathrm{l})$ were able to induce RhoA upregulation; however, the extent of the increase was lower in the mannitol groups than in the high salt medium groups (115 and $137 \mathrm{mmol} / \mathrm{l})$. These results indicated that, in addition to osmotic pressure, salt is the main driving force associated with the upregulation of RhoA expression and activity. PRMT-1 expression was unaffected by an increase in mannitol, thus excluding the possibility that the gene alterations were induced by osmolality.

The present study knocked down PRMT-1 expression using siRNA, in order to explore the role of ADMA in high salt-mediated RhoA/ROCK activation and eNOS inhibition. PRMTs are a family of proteins that either monomethylate or dimethylate the guanidino nitrogen atoms on arginine side chains. PRMT-1 is the predominant type I PRMT in mammalian cells, which catalyzes arginine residues and facilitates the formation of ADMA residues (12). Aberrant PRMT expression has been reported to contribute to the pathogenesis of pulmonary diseases (33). The present study demonstrated that PRMT-1-specific siRNA attenuated high salt-induced increases in ADMA secretion, confirming that PRMT-1 is one of the key upstream regulators of ADMA secretion, and that the knockdown of PRMT-1 is sufficient to decrease ADMA secretion. Via knockdown of PRMT-1, the levels of ADMA could be manipulated, and the hypotheses that ADMA has an important role in high salt-induced RhoA pathway activation and eNOS inhibition could be verified. The results of the present study indicated that downregulation of ADMA was able to inhibit the upregulated expression and activity of RhoA and the expression of eNOS. Therefore, ADMA secretion and RhoA/ROCK activation may exert synergistic effects on eNOS expression in response to high salt medium.

In conclusion, the results of the present study indicated that high salt medium can increase ADMA secretion, activate RhoA activity and inhibit eNOS expression. Furthermore, ADMA was revealed to have a crucial role in the mediation of these effects. The present study was the first, to the best of our knowledge, to identify a mechanism and provide information regarding how high salt intake induces endothelial dysfunction in vitro. PRMT-siRNA may be considered as a novel gene therapy used to reduce ADMA production. The present study provided a novel theoretical basis for the prevention and treatment of salt-sensitive hypertension.

\section{Acknowledgements}

The present study was supported by funds from the Nature Science Foundation of China (grant no. 30900616); the National Program on Key Basic Research Project of China (973 Program; grant no. 2012CB517804); the Department of Cardiovascular Medicine, First Affiliated Hospital of Medical College of Xi'an Jiaotong University; the Institute of Cardiovascular Channelopathy, Key Laboratory of Environment and Genes Related to Diseases (Xi'an Jiaotong University), Ministry of Education; and the Key Laboratory of Molecular Cardiology, Shannxi Province.

\section{References}

1. Miyoshi A, Suzuki H, Fujiwara M, Masai M and Iwasaki T: Impairment of endothelial function in salt-sensitive hypertension in humans. Am J Hypertens 10: 1083-1090, 1997.

2. Félétou M and Vanhoutte PM: Endothelial dysfunction: A multifaceted disorder (The Wiggers Award Lecture). Am J Physiol Heart Circ Physiol 291: H985-H1002, 2006.

3. Davignon J and Ganz P: Role of endothelial dysfunction in atherosclerosis. Circulation 109 (23 Suppl 1): III27-III32, 2004.

4. Förstermann U and Münzel T: Endothelial nitric oxide synthase in vascular disease: From marvel to menace. Circulation 113: 1708-1714, 2006

5. Bragulat E and de la Sierra A: Salt intake, endothelial dysfunction, and salt-sensitive hypertension. J Clin Hypertens (Greenwich) 4: 41-46, 2002.

6. Toda N and Arakawa K: Salt-induced hemodynamic regulation mediated by nitric oxide. J Hypertens 29: 415-424, 2011.

7. Schmidlin O, Forman A, Leone A, Sebastian A and Morris RC Jr: Salt sensitivity in blacks: Evidence that the initial pressor effect of $\mathrm{NaCl}$ involves inhibition of vasodilatation by asymmetrical dimethylarginine. Hypertension 58: 380-385, 2011.

8. Riento K and Ridley AJ: Rocks: Multifunctional kinases in cell behaviour. Nat Rev Mol Cell Biol 4: 446-456, 2003.

9. Kobayashi N, Takeshima H, Fukushima H, Koguchi W, Mamada Y, Hirata H, Machida Y, Shinoda M, Suzuki N, Yokotsuka F, et al: Cardioprotective effects of pitavastatin on cardiac performance and remodeling in failing rat hearts. Am J Hypertens 22: 176-182, 2009.

10. Loirand G and Pacaud P: The role of Rho protein signaling in hypertension. Nat Rev Cardiol 7: 637-647, 2010.

11. Laufs U and Liao JK: Post-transcriptional regulation of endothelial nitric oxide synthase mRNA stability by Rho GTPase. J Biol Chem 273: 24266-24271, 1998.

12. Tang J, Frankel A, Cook RJ, Kim S, Paik WK, Williams KR, Clarke S and Herschman HR: PRMT1 is the predominant type I protein arginine methyltransferase in mammalian cells. J Biol Chem 275: 7723-7730, 2000.

13. Fiedler L and Wojciak-Stothard B: The DDAH/ADMA pathway in the control of endothelial cell migration and angiogenesis. Biochem Soc Trans 37: 1243-1247, 2009. 
14. Li XH,Peng J, Tan N, Wu WH,Li TT, Shi RZ and Li YJ: Involvement of asymmetric dimethylarginine and Rho kinase in the vascular remodeling in monocrotaline-induced pulmonary hypertension. Vascul Pharmacol 53: 223-229, 2010.

15. Fiedler L: The DDAH/ADMA pathway is a critical regulator of NO signalling in vascular homeostasis. Cell Adh Migr 2: 149-150, 2008

16. Wojciak-Stothard B, Torondel B, Zhao L, Renné T and Leiper JM: Modulation of Racl activity by ADMA/DDAH regulates pulmonary endothelial barrier function. Mol Biol Cell 20: 33-42, 2009.

17. de Wardener HE, He FJ and MacGregor GA: Plasma sodium and hypertension. Kidney Int 66: 2454-2466, 2004.

18. He FJ, Markandu ND, Sagnella GA, de Wardener HE and MacGregor GA: Plasma sodium: Ignored and underestimated. Hypertension 45: 98-102, 2005.

19. Orlov SN and Mongin AA: Salt-sensing mechanisms in blood pressure regulation and hypertension. Am J Physiol Heart Circ Physiol 293: H2039-H2053, 2007.

20. Oberleithner H, Kusche-Vihrog K and Schillers H: Endothelial cells as vascular salt sensors. Kidney Int 77: 490-494, 2010.

21. Livak KJ and Schmittgen TD: Analysis of relative gene expression data using real-time quantitative PCR and the 2(-Delta Delta C(T)) Method. Methods 25: 402-408, 2001.

22. van Nieuw Amerongen GP and van Hinsbergh VW: Cytoskeletal effects of Rho-like small guanine nucleotide-binding proteins in the vascular system. Arterioscler Thromb Vasc Biol 21: 300-311, 2001.

23. Fang Y, Mu JJ, He LC, Wang SC and Liu ZQ: Salt loading on plasma asymmetrical dimethylarginine and the protective role of potassium supplement in normotensive salt-sensitive Asians. Hypertension 48: 724-729, 2006

24. Fujiwara N, Osanai T, Kamada T, Katoh T, Takahashi K and Okumura K: Study on the relationship between plasma nitrite and nitrate level and salt sensitivity in human hypertension: Modulation of nitric oxide synthesis by salt intake. Circulation 101: 856-861, 2000.
25. Zhou MS, Adam AG, Jaimes EA and Raij L: In salt-sensitive hypertension, increased superoxide production is linked to functional upregulation of angiotensin II. Hypertension 42: 945-951, 2003.

26. Taylor NE, Maier KG, Roman RJ and Cowley AW Jr: NO synthase uncoupling in the kidney of Dahl S rats: Role of dihydrobiopterin. Hypertension 48: 1066-1071, 2006.

27. Yang T, Zhang A, Honeggar M, Kohan DE, Mizel D, Sanders K, Hoidal JR, Briggs JP and Schnermann JB: Hypertonic induction of COX-2 in collecting duct cells by reactive oxygen species of mitochondrial origin. J Biol Chem 280: 34966-34973, 2005.

28. Zhang Z, Dmitrieva NI, Park JH, Levine RL and Burg MB: High urea and $\mathrm{NaCl}$ carbonylate proteins in renal cells in culture and in vivo, and high urea causes 8-oxoguanine lesions in their DNA. Proc Natl Acad Sci USA 101: 9491-9496, 2004.

29. Zhou X, Ferraris JD and Burg MB: Mitochondrial reactive oxygen species contribute to high $\mathrm{NaCl}$-induced activation of the transcription factor TonEBP/OREBP. Am J Physiol Renal Physiol 290: F1169-F1176, 2006.

30. Sydow K and Münzel T: ADMA and oxidative stress. Atheroscler Suppl 4: 41-51, 2003

31. Di Ciano-Oliveira C, Sirokmány G, Szászi K, Arthur WT, Masszi A, Peterson M, Rotstein OD and Kapus A: Hyperosmotic stress activates Rho: Differential involvement in Rho kinase-dependent MLC phosphorylation and NKCC activation. Am J Physiol Cell Physiol 285: C555-C566, 2003.

32. Malek AM, Xu C, Kim ES and Alper SL: Hypertonicity triggers RhoA-dependent assembly of myosin-containing striated polygonal actin networks in endothelial cells. Am J Physiol Cell Physiol 292: C1645-C1659, 2007.

33. Zakrzewicz D, Zakrzewicz A, Preissner KT, Markart P and Wygrecka M: Protein arginine methyltransferases (PRMTs): Promising targets for the treatment of pulmonary disorders. Int J Mol Sci 13: 12383-12400, 2012. 